\title{
GPX-Macrophage Expression Atlas: A database for expression profiles of macrophages challenged with a variety of pro-inflammatory, anti-inflammatory, benign and pathogen insults Graeme R Grimes*, Stuart Moodie, John S Beattie, Marie Craigon, Paul Dickinson, Thorsten Forster, Andrew D Livingston, Muriel Mewissen, Kevin A Robertson, Alan J Ross, Garwin Sing and Peter Ghazal*
}

Address: The Scottish Centre for Genomic Technology and Informatics, University Of Edinburgh, 49 Little France Crescent, Edinburgh, EH16 4SB, UK

Email: Graeme R Grimes* - Graeme.Grimes@ed.ac.uk; Stuart Moodie - stuart.moodie@ed.ac.uk; John S Beattie - john.beattie@ed.ac.uk; Marie Craigon - marie.craigon@ed.ac.uk; Paul Dickinson - Paul.Dickinson@ed.ac.uk; Thorsten Forster - thorsten.forster@ed.ac.uk; Andrew D Livingston - A.D.Livingston@sms.ed.ac.uk; Muriel Mewissen - Muriel.Mewissen@ed.ac.uk;

Kevin A Robertson - Kevin.Robertson@ed.ac.uk; Alan J Ross - Alan.J.Ross@ed.ac.uk; Garwin Sing - garwin.sing@ed.ac.uk; Peter Ghazal* - p.ghazal@ed.ac.uk

* Corresponding authors

Published: 12 December 2005

BMC Genomics 2005, 6:178 doi:10.1186/147|-2164-6-178
Received: II July 2005

Accepted: 12 December 2005

This article is available from: http://www.biomedcentral.com//47/-2/64/6/I78

(c) 2005 Grimes et al; licensee BioMed Central Ltd.

This is an Open Access article distributed under the terms of the Creative Commons Attribution License (http://creativecommons.org/licenses/by/2.0), which permits unrestricted use, distribution, and reproduction in any medium, provided the original work is properly cited.

\begin{abstract}
Background: Macrophages play an integral role in the host immune system, bridging innate and adaptive immunity. As such, they are finely attuned to extracellular and intracellular stimuli and respond by rapidly initiating multiple signalling cascades with diverse effector functions. The macrophage cell is therefore an experimentally and clinically amenable biological system for the mapping of biological pathways. The goal of the macrophage expression atlas is to systematically investigate the pathway biology and interaction network of macrophages challenged with a variety of insults, in particular via infection and activation with key inflammatory mediators. As an important first step towards this we present a single searchable database resource containing high-throughput macrophage gene expression studies.
\end{abstract}

Description: The GPX Macrophage Expression Atlas (GPX-MEA) is an online resource for gene expression based studies of a range of macrophage cell types following treatment with pathogens and immune modulators. GPX-MEA follows the MIAME standard and includes an objective quality score with each experiment. It places special emphasis on rigorously capturing the experimental design and enables the searching of expression data from different microarray experiments. Studies may be queried on the basis of experimental parameters, sample information and quality assessment score. The ability to compare the expression values of individual genes across multiple experiments is provided. In addition, the database offers access to experimental annotation and analysis files and includes experiments and raw data previously unavailable to the research community.

Conclusion: GPX-MEA is the first example of a quality scored gene expression database focussed on a macrophage cellular system that allows efficient identification of transcriptional patterns. The resource will provide novel insights into the phenotypic response of macrophages to a variety of benign, inflammatory, and pathogen insults. GPX-MEA is available through the GPX website at http://www.gti.ed.ac.uk/GPX. 


\section{Background}

Macrophages are one of the most intensively studied cells of the immune system. They have been shown to play a key role in both the innate and adaptive immune response and are capable of responding to a range of signals leading to classical or alternative activation of immune responses which may be classified as innate, cellular, or humoral, [1]. Their function as a mobile unicellular system make them an ideal biological model for taking a systems perspective to pathway analysis, and in silico models of the macrophage cell and signalling pathway are beginning to emerge [2]. Clinically, macrophages are increasingly recognised as key determinants of a wide range of diseases including cancer, atherosclerosis, infection, inflammation and auto-immunity. Because of the broad spectrum of biological processes and pathological conditions involving macrophages, studies and data on macrophage biology are highly heterogeneous and dispersed. There is an emerging need to integrate diverse studies and provide highly reliable datasets for systems level analysis in this cell system. At the Scottish Centre for Genomic Technology and Informatics (GTI), we have developed the GPX-Macrophage Expression Atlas (GPXMEA). The GTI is a founder member of the Integrated Macrophage Biology Group (IMBG), which is a network of institutes with interests in a wide variety of macrophage related research topics, such as inflammation, infectious disease, cancer biology and immunity. Notably, all members of the IMBG have a common interest in elucidating key pathways within the macrophage and the network allows researchers to contribute a variety of biological knowledge and experimental and computational techniques to this area.

GPX-MEA is a gene expression database that stores MIAME-compliant [3] microarray experiments and associated data from a variety of human and murine macrophage cell types following treatment with pathogens and immune modulators. This was accomplished by the collation of macrophage microarray studies from a diverse group of private and public resources followed by validation and curation into a central database. GPX-MEA complements other larger gene expression repositories such as GEO [4] and ArrayExpress [5] by providing focussed datasets and comparative facilities for macrophage biologists, immunologists and those interested in systems biology. This enables the researcher for the first time to rapidly search gene expression values from a number of experiments involving different macrophage types, under diverse conditions within a single resource. Furthermore, GPX-MEA provides a coherent group of annotated raw analysis files that can be used by the computational biologist for systems level analysis.
A major challenge when using microarray data to elucidate molecular pathways is the development of methods for the reliable comparison of results from different studies. This issue is further compounded by the fact that microarray assays are highly sensitive to technical perturbations and the lack of standard units of gene expression. It has been shown that microarray experiments, even using the same platform, can vary greatly between groups and laboratories [6]. In an attempt to address this issue in GPX-MEA, a quality assessment score for each experiment in the database is derived using defined criteria to give users an indication of the underlying quality of the experiment design, quality control and data structures. This score can also be used to retrieve experiments based on their quality requirements using the GPX-MEA web interface.

Here we report on the data curation process, quality assessment, content, search facilities and integration tools that are components of the GPX-MEA.

\section{Construction and content}

\section{Construction}

GPX-MEA is part of the GPX database system at the Scottish Centre for Genomic Technology and Informatics (GTI, http://www.gti.ed.ac.uk). The GPX system comprises two MIAME-compliant microarray databases, GPX Repository and GPX Discovery. The data is first curated into GPX Repository, a biochip Laboratory Information Management System developed at the GTI. GPX Repository is designed around a set of web enabled data entry forms that assist in the data capture process ensuring that MIAME-compliant information is obtained.

On completion of curation, experiments are migrated from GPX Repository to GPX Discovery. GPX Discovery has been designed with web interfaces which allow the user to query and retrieve data within GPX-MEA. It also has the additional functionality of being able to retrieve and compare individual probe signal intensity values across experiments.

The underlying database schema of GPX Discovery, maxdSQL-Plus, is an enhanced version of maxdSQL 1.2 (maxd, http://bioinf.man.ac.uk/microarray/maxd/). Enhancement to the maxdSQL schema include extensions to store image analysis files and annotations from the NCBI UniGene database [7].

Data within the GPX-MEA is accessed through a webbased user interface developed at the GTI and implemented using Perl and an Apache-mod_perl server. The web application interfaces with an Oracle 9iR2 relational database management system to search and retrieve the experimental data. The schema, scripts, tools and docu- 
mentation are available at http://www.gti.ed.ac.uk/GPX/ software.

\section{Data acquisition}

GPX-MEA contains gene expression data from microarray experiments conducted on a variety of human and murine macrophage cell types challenged with pro-inflammatory, anti-inflammatory, benign and pathogen insults.

Microarray studies fulfilling these biological criteria were collated from a survey of five major gene expression repositories (GEO [4], ArrayExpress [5], SMD[8], RAD [9] and CIBEX [10]). In addition to experiments submitted to public microarray repositories, GPX-MEA includes experiments and associated data selected from a survey of over 2500 publications in the field of macrophage immunology and infection. This combined approach to data collation was adopted in an attempt to consolidate data which had previously been scattered among a variety of repositories and laboratories.

The investigators of each experiment selected for inclusion in the database were contacted and asked to supply raw image and image analysis files (Affymetrix DAT and CEL files), and any MIAME information that were not reported in the original publication or database entry. Responses were received from eleven of the initial eighteen investigators contacted to supply data and information to GPX-MEA.

In addition to experimental information and data obtained from the public domain, GPX-MEA also contains original unpublished microarray experiments conducted at the GTI. These experiments characterise the gene expression profile of murine bone marrow derived macrophages grown under standard and serum free growth conditions. Information and associated data files for these and other experiments may be retrieved from GPX-MEA using the GPX Discovery web interface.

\section{Data curation}

Data submitted to the GPX-MEA has followed a manual curation process. At present, subjective evaluation criteria are employed during the quality assessment of microarray data. As a result, expert manual curation was undertaken to ensure the value and biological relevance of information held within the GPX-MEA. Efforts in the future will be directed to methods for the automation of this process.

The curation process follows standard operating procedures that include the assessment of the experiment data against defined criteria and validation of experiment information against MIAME standards.
For this first public release of GPX-MEA, studies conducted using the Affymetrix GeneChip ${ }^{\circledast}$ platform have been preferentially selected since they have widely recognised quality control parameters, use of standardized manufacturing and experimental protocols and familiarity of the platform with the biological and computational users. Future releases of GPX-MEA will contain experiments hybridized using spotted custom array platforms and other proprietary platforms, such as those from Agilent and GE Healthcare.

Before submission to the database, analysis files were all processed using the same software program and normalisation algorithm. Affymetrix DAT files were processed using Affymetrix-Gene Chip Operating System (GCOS). The CHP files were generated from CEL files using Affymetrix Global scaling normalisation to a Target Intensity Value of 100 (TGT-100). In some instances DAT files were unavailable and the original CEL files were used to generate the CHP files. The use of this global normalization algorithm provides a uniform measure which allows a preliminary comparison of expression values across experiments within the databases. Other values that are stored within the database from the CHP files are the detection call $(\mathrm{P}, \mathrm{M}, \mathrm{A})$ and the $\mathrm{p}$-value of detection.

\section{Quality assessment}

GPX-MEA contains experiments of varying complexity and experimental design collated from a variety of resources. To take into account the variations in quality and statistical power of experiments a GTI-derived ordinal scale quality assessment score is assigned to each experiment added to the database. The score enables the user to make an informed judgement on the reliability of results obtained from experiments within GPX-MEA and allows for experiment selection based on quality requirements. It also functions to provide a metric for systems biologist interested in integrating data sets from the database.

The quality assessment score ranges from 0 (low) to 28 (high) and is derived from six defined categories each with a 3-point $(0,1,2)$ scale. The score is also stored as a percentage of the maximum attainable value within the database. Categories are also weighted by relative importance of the assessment on a 3-point $(1,2,3)$ scale. The scoring categories are as follows: use of consistent standard operating procedures (SOPs) [11], sample quality control, the number of biological replicates in the study, and finally, variation levels and data distributions [1214]. All scoring categories, scoring levels and weightings are pre-determined (Table 1) and can be viewed using the GPX Discovery interface. Categories and assessment tools were selected after extensive discussion between biological investigators, statisticians and bioinformaticians. This process was informed by the GTI's experience in high 
Table I: Quality Assessment Criteria for GPX-MEA

\begin{tabular}{|c|c|c|c|}
\hline Assessment category & Tool & Weight & (Score)Criteria \\
\hline $\begin{array}{l}\text { Standard Operating Procedures } \\
\text { (SOPs) }\end{array}$ & Experiment description/protocol & 2 & $\begin{array}{l}2 \text { - Standard Operating Procedures have been documented } \\
\text { and followed. } \\
\text { I - Only partial SOPs have been documented applied or } \\
\text { followed } \\
0 \text { - SOPs not known or not followed }\end{array}$ \\
\hline Sample size & Experiment description/protocol & 3 & $\begin{array}{l}2 \text { - Number of independent biological samples exceeds } 5 \text { per } \\
\text { group, allowing for reasonably high statistical power in the } \\
\text { hypothesis testing. } \\
\text { I- } 3-5 \text { independent biological samples per group, allowing to } \\
\text { apply minimum level of statistics } \\
0-\text { I or } 2 \text { independent biological samples per group (e.g. } \\
\text { pilot experiment), reducing the applicability of statistics. }\end{array}$ \\
\hline Image quality & $\begin{array}{l}\text { Image scan or numerical } \\
\text { representation (Affymetrix) }\end{array}$ & 3 & $\begin{array}{l}2 \text { - No signs of chip defects, background noise (RawQ) } \\
\text { within } 5 \text { point range of each other. Scaling factor within I-2 } \\
\text { fold range. } \\
\text { I - Isolated chip defects, RawQ within } 5 \text { point range, scaling } \\
\text { factor I-3 } \\
0 \text { - Systematic chip defects, saturation, RawQ outside } 5 \\
\text { point range, scaling factor greater than } 3 \text {. }\end{array}$ \\
\hline Target sample quality & $\begin{array}{l}\text { Electropherogram, UV- } \\
\text { Spectrophotometer }\end{array}$ & I & $\begin{array}{l}2 \text { - Presence of } 18 S \text { and } 28 S \text { ribosomal subunits with no sign } \\
\text { of degradation in any sample (RNA integrity). Absorbance } \\
\text { ratio of A260/280 close to } 2 \text { (RNA purity) [16]. I - Either } \\
\text { RNA integrity or purity criteria are met. } 0-\text { Neither } \\
\text { integrity nor purity criteria are met, or sample quality } \\
\text { assessment was not performed, or no information provided. }\end{array}$ \\
\hline $\begin{array}{l}\text { Sample level data variation/ } \\
\text { distribution }\end{array}$ & $\begin{array}{l}\text { Box-and-whisker plots, supporting } \\
\text { MA plots if required. }\end{array}$ & 3 & $\begin{array}{l}2 \text { - Very consistent array medians and Inter-Quartile-Ranges } \\
\text { across all samples before normalisation procedures (note: } \\
\text { underlying assumption is that any treatments/conditions } \\
\text { should not cause differential expression in more than 5-I0\% } \\
\text { of all gene probes on the array.) I - Very small number of } \\
\text { inconsistent data distributions with assumptions of a } \\
\text { correctable difference in array signal intensity met (all genes } \\
\text { on array affected, inter-array gene relationships are linear or } \\
\text { global differences are expected, e.g. LPS treatment). } 0- \\
\text { Inconsistent median signal levels and spread (IQR) of data } \\
\text { throughout the experiment, no biological explanation given. }\end{array}$ \\
\hline Gene level data variation & $\begin{array}{l}\text { Coefficient of Variation versus } \\
\text { Mean Expression plots }\end{array}$ & 2 & $\begin{array}{l}2 \text { - Coefficient of Variation for majority of genes across all } \\
\text { replicates within a sample group is less than } 20 \% \text {. Genes } \\
\text { above this CV level are mostly in the lower signal range (due } \\
\text { to measurement error). I - A small proportion of all genes } \\
\text { has higher } \mathrm{CV} \text { values than } 20 \% \text {, with a small number of these } \\
\text { in the medium to high expression range. } 0-\mathrm{A} \text { large } \\
\text { proportion of genes has a CV above } 20 \% \text { across most of the } \\
\text { expression range. }\end{array}$ \\
\hline
\end{tabular}

A quality control assessment is made for each experiment curated into GPX-MEA. The quality assessment score is calculated using quantitative and qualitative scores in different areas; use of Standard Operating Procedures, the number and use of independent biological replicates, quality of image, sample quality control and structure of numeric data (spread, reproducibility). A combination of the different areas is used to give a total quality assessment score. This score can be used to indicate the quality of the underlying experiment in the database and as such is subject to interpretation. Where data is unavailable for assessment no score is assigned.

throughput microarray experimentation and took in consideration published literature[11,12,14-18]. Initially an additional assessment category was used that assigned a score to the biological relevance of the experiment. This criterion was omitted from the final assessment system so that the score was focussed on technical sources of variation in the study. This resulted in a more reproducible scoring methodology.

It is worth noting that given the complexity and variety of experimental designs and data structures, it is not feasible to develop fully objective criteria based on non-arbitrary threshold values, instead we have sought to provide the user with a graded indication of the underlying quality of an experiment with a scoring system that is reproducible.

The first step in scoring an experiment is to obtain MIAME compliant information about an experiment's design and implementation. Using this information the score for SOPs, sample size and sample quality control can be assigned. The next criterion assesses image files and includes analysis of the background noise (RAWQ) and 
Table 2: Summary of microarray experiment in GPX-MEA.

\begin{tabular}{|c|c|c|c|c|c|c|}
\hline Accession & Experimental Factors & $\begin{array}{l}\text { Host } \\
\text { Species }\end{array}$ & Cell types & $\begin{array}{l}\text { Hybridisations } \\
\text { Count }\end{array}$ & Array Type & Reference \\
\hline GPX-000032 & time, compound, genotype & Mouse & BMDMs & 17 & MG_U74Av2 & {$[20]$} \\
\hline GPX-000034 & time, infection, compound & Mouse & BMDMs & 12 & MG_U74Av2 & [21] \\
\hline GPX-000035 & compound, genotype & Mouse & BMDMs & 12 & MG_U74Av2 & {$[21]$} \\
\hline GPX-000036 & infection, cell type & Human & $\begin{array}{l}\text { Dendritic cells, monocyte derived } \\
\text { macrophages }\end{array}$ & 28 & HG_U95Av2 & {$[26]$} \\
\hline GPX-000037 & compound, protocol & Human & PBMCs & 12 & HG_U95Av2 & {$[24]$} \\
\hline GPX-000038 & compound, protocol & Human & PBMCs & 18 & HG_U95Av2 & $\mathrm{N} / \mathrm{A}$ \\
\hline GPX-000039 & time, compound & Mouse & RAW264.7 & 3 & MOE430A & {$[25]$} \\
\hline GPX-000040 & time, compound, genotype & Mouse & BMDMs & 10 & MG_U74Av2 & {$[23]$} \\
\hline GPX-00004I & infection & Mouse & Monocyte derived macrophages & 30 & MG_U74Av2 & {$[22]$} \\
\hline GPX-000043 & protocol & Mouse & BMDMs & 6 & MG_U74Av2 & $\mathrm{N} / \mathrm{A}$ \\
\hline GPX-000045 & protocol & Mouse & BMDMs & 4 & MG_U74Av2 & $N / A$ \\
\hline
\end{tabular}

BMDMs indicate cells are bone marrow derived macrophages. PBMCs indicate cells are peripheral blood mononuclear cells. N/A indicates experiments unpublished.

variation in mean signal intensity (Scaling Factor) for the arrays. The final criterion seeks to measure sample level variation and gene level variation from visualizations output using $\mathrm{R}$ and Bioconductor[19] software from the raw image analysis files. Scores are assigned to each experiment by the curator based on the criteria in Table 1 .

To illustrate the assessment method the scoring of the category "sample size" is described. In the category "sample size", the number of biological replicates per group is determined and scored using the criteria in table 1 . In the case of an experiment with 3 biological replicates per group a mid score of 1 is assigned. After the initial score is calculated, it is adjusted by relative importance using the weighting of the category. The sample size category is deemed of high importance $[15,17,18]$ and is given a weighting of 3 , therefore the initial score of 1 is multiplied by the category weighting 3 , and assigned a value of 3 .

A record detailing the score in each category for an individual experiment can be viewed using GPX-MEA. Further, the combined overall percentage score can be used to select and retrieve experiments using the GPX Discovery interface.

Future versions of GPX-MEA will incorporate nonAffymetrix experiments. This will require modifications to the scoring system which are likely to include; more stringent requirements for the numbers of biological replicates used in custom arrays based experiments, due to the larger noise variation, the use of different visualisation techniques to assess quality and an additional criterion to address the suitability of the reference sample.

\section{Data content}

The current release of GPX-MEA (May 2005) consists of 11 public microarray experiments with a total of 152 hybridizations from 3 Affymetrix GeneChip ${ }^{\oplus}$ platforms (Table 2). Eight of the eleven experiments have an associated literature publication [20-26]. These experiments include the treatment of human or murine macrophages with a range of agents including viral infection (e.g. murine cytomegalovirus), treatment with cytokines (e.g. IFN-alpha, IFN-beta, IFN-gamma, IL-4, IL-6) and serum withdrawal. In addition, the effects of these treatments had been examined using a range of macrophage genotypes in the mouse (wild type, PIAS-/-, IRF1-/-, SOCS3-/-).

At present, GPX-MEA has a further 12 MIAME-compliant experiments with a total of 246 hybridizations stored in a restricted access site. This allows the accommodation of unpublished microarray experiments. Experiments within this access controlled site will be made publicly accessible following scientific publication. Further macrophage gene expression studies have been identified for deposition in the GPX-MEA database and routine updates to the database are scheduled to include emerging research findings.

\section{Utility}

Search pages

Users can browse and retrieve data from GPX-MEA using the user oriented GPX Discovery web based graphical interface http://www.gti.ed.ac.uk/GPX. Searches can be performed in three different sections of the interface; experiments, arrays and probes. A search is made using a number of separate criteria such as accession number, technology type, target organism, quality assessment score or keywords. For example, all microarray experiments conducted using humans as the source organism can be retrieved by performing a search selecting Homo sapiens from the organism search field. The results of a query are displayed in a table with hyperlinks to the corresponding experiment, array or probe record. 


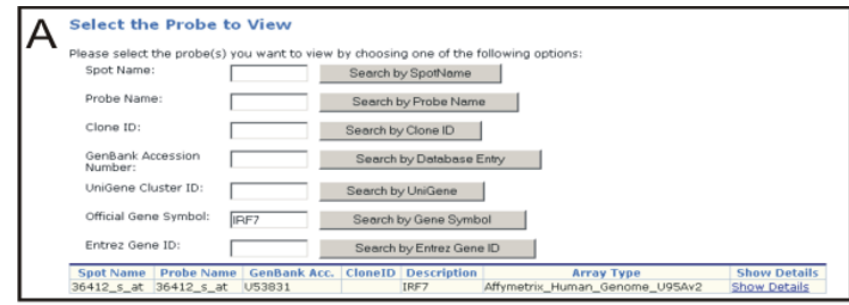

B Probe: 36412_s_at.

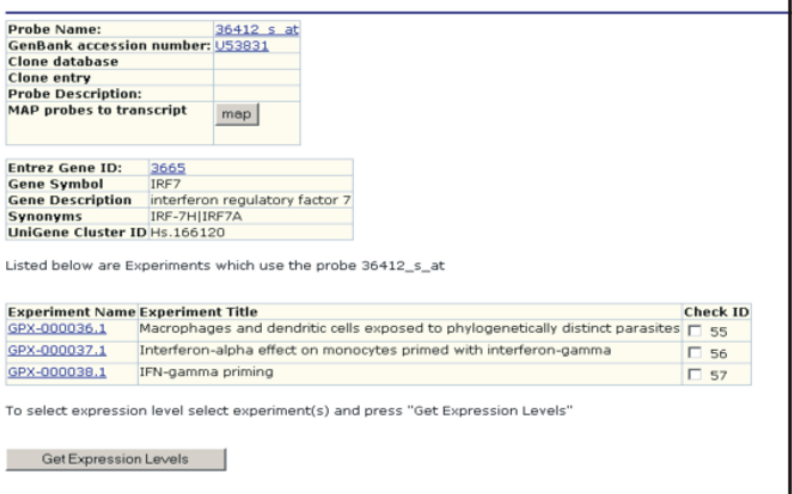

Macrophages and dendritic cells exposed to phylogenetically distinct parasites
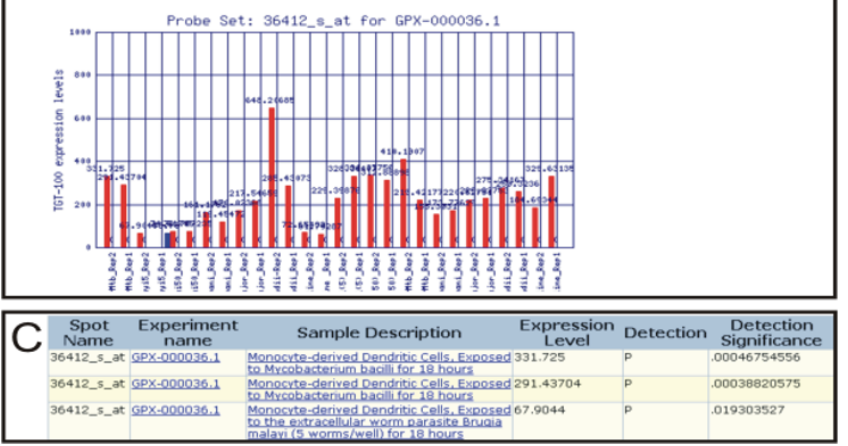

Figure I

Comparison of the expression profile of IRF7 across three experiments within GPX-MEA. (A) From the probe search interface the user searches for the gene 'IRF7'. (B) From the probe record the user selects the experiments they wish to view expression values from. (C) A list of expression values for the probe is displayed alongside the measurement and experiment name.

\section{Experiment}

Experiments are defined in GPX-MEA as the complete set of bioassays (hybridizations) and their descriptions for a common purpose, following Microarray Gene Expression Data Society (MGED) ontology specifications. Once an experiment has been selected the corresponding database entry is displayed. An experiment record shows the provenance, experimental design, protocols, sample information, array details, quality assessment and a list of array measurements. Expression values can be selected from the experiment record by selecting the desired measurement records. Measurement data are presented to the users as a comma separated value (CSV) file. Significantly, the user can also obtain original image analysis files, e.g. the Affymetrix CEL files, from the database providing additional flexibility for an extended analysis. Access to the original image analysis files is an especially valuable resource for those interested in computational analysis and data integration.

\section{Array type}

The array type section allows the user to browse the list of array types stored within the database. An array type record displays the description of the array type and the manufacturer. The array layout with probe annotation obtained from a local copy of the UniGene database can also be downloaded as a CSV file from a link within a record.

\section{Probe}

The probe section allows the user to search and retrieve individual probe details from the database based on criteria such as Entrez Gene ID, Gene Symbol and UniGene ID. Once a probe record is selected the user can view probe annotation within the record and by following links to external databases, Adapt[27], NetAffx [28] and NCBI Gene[29]. The probe record also displays a list of experiments associated with the probe. Using the list of associated experiments, the user can compare the gene expression levels for that probe across the GPX-MEA. For example (Fig 1), the user searches for probes that target the gene interferon regulatory factor 7 using the gene symbol 'IRF7'. From the returned results the user selects a probe record to view more details. From this probe record, the user then selects the experiments within the database to compare the gene expression levels. The results are formatted as a table containing signal intensities, Affymetrix detection calls $(\mathrm{P}, \mathrm{M}, \mathrm{A})$ and $\mathrm{p}$-value of detections for every measurement from the selected experiments. The signal intensity of Affymetrix probe sets are the TGT-100 normalised signal. The use of this consistent global normalisation algorithm within the database allows for a preliminary comparison of probe expression levels between the distinct microarray experiments.

\section{Biolink MatchMaker}

The Probe section provides a link to the BioLink MatchMaker integration tool which enables the comparison of different array types. BioLink MatchMaker uses NCBI's UniGene Cluster IDs to identify probes which represent equivalent genes on two different microarray platforms of the same species stored within GPX-MEA. Users can also submit a list of up to 1000 GenBank or UniGene ID to identify probe matches with any array type in the database using the Batch query function. Results can be displayed either as HTML tables containing hyperlinks to the corre- 
sponding UniGene entry or can be downloaded as a CSV file for further analysis. For example, the Affymetrix GeneChips ${ }^{\circledR}$ MG U74Av2 and MOE430A version 2 can be compared using BioLink MatchMaker to find probes that share equivalent genes. The results show that there are 7454 unique probe matches between the two platforms. The user can then view the results in a separate webpage or download the results for further analysis.

\section{Future work}

GPX-MEA is under active development and will continue to collate macrophage expression studies through online database searches and collaboration with principle investigators. This data will be assessed and manually curated within the GPX-MEA and released in routine database updates. In addition, experiments that profile the macrophage response to infection and immune regulators conducted within GTI will also be included in the database. There will also be continual development of curation and quality assessment protocols to maintain the high standard of experimental description and data within the database.

New analytical tools in development for the GPX-MEA include the ability to query gene expression using interactive immunological pathway maps and an automated statistical analysis tool to guide the user through normalisation and analysis processes. The export of MAGE-ML (Microarray Gene Expression-Markup language) from the database is also being developed so that data may be shared between other public repositories and users of microarray data. In addition, GPX is part of the Edinburgh National Translational Cancer Research Network Centre resources (NTRAC) and relevant work emerging from this project will be included in GPX-MEA.

\section{Conclusion}

Here we present GPX-MEA, a MIAME compliant online database that exploits and captures the wealth of expression studies related to macrophage biology data in a centralised resource. Experiments are assessed and submitted to the database by expert curators and includes some information and data, such as image analysis files, that were not previously publicly available.

This is the first example of a focussed macrophage gene expression database that allows efficient identification of gene expression patterns in this key player in the host immune system.

GPX-MEA also provides highly reliable datasets with associated contextual information that can be used by the computational biologist for systems level analysis. In addition, it also utilises a unique quality assessment scor- ing system that enables the user to select experiments based on their quality requirements.

\section{Availability}

GPX-MEA is publicly available and can be accessed at http://www.gti.ed.ac.uk/GPX using web browsers. The GPX Discovery software is available for download at http://www.gti.ed.ac.uk/GPX/software.

\section{Authors' contributions}

GG carried out the data collation, curation, and quality assessment and drafted the manuscript. SM and GG carried out the database development, design and data migration. $\mathrm{TF}, \mathrm{KR}, \mathrm{PD}, \mathrm{MC}$ participated in the design of the quality control criteria. $\mathrm{GS}, \mathrm{AL}, \mathrm{MC}$, AR contributed data to the database. PG and JB conceived of the database, and participated in its design and coordination. PG, JB, $\mathrm{TF}, \mathrm{KR}, \mathrm{PD}, \mathrm{SM}$ and MM helped to draft the manuscript. All authors read and approved the final manuscript.

\section{Acknowledgements}

This work was supported by the Scottish Higher Education Funding Council, Biotechnology and Biological Sciences Research Council, Wellcome Trust, the European Regional Development Fund and the INFOBIOMED Network of Excellence (European Commission FP6 Programme: contract number IST-2002-507585). We thank our colleagues at the GTI and collaborators Liu Bin, Ke Shuai, Reinhard Hoffmann, Xiaoyu Hu, Rachael Zoë

Murray, Jenny Stow, Thomas Nutman, Damien Chaussabel, Daniel Popkin, Skip Virgin and loannis Tassiulas for contributing to this resource.

\section{References}

I. Gordon S: Alternative activation of macrophages. Nat Rev Immunol 2003, 3(I):23-35.

2. Oda K, Kimura T, Matsuoka Y, Funahashi A, Muramatsu M, Kitano H: Molecular Interaction Map of a Macrophage. AfCS Research Reports 2004, 2( I 4): I- 12.

3. Brazma A, Hingamp P, Quackenbush J, Sherlock G, Spellman $P$, Stoeckert C, Aach J, Ansorge W, Ball CA, Causton HC, Gaasterland T, Glenisson P, Holstege FC, Kim IF, Markowitz V, Matese JC, Parkinson H, Robinson A, Sarkans U, Schulze-Kremer S, Stewart J, Taylor R, Vilo J, Vingron M: Minimum information about a microarray experiment (MIAME)-toward standards for microarray data. Nat Genet 200I, 29(4):365-37I.

4. Barrett T, Suzek TO, Troup DB, Wilhite SE, Ngau WC, Ledoux P, Rudnev D, Lash AE, Fujibuchi W, Edgar R: NCBI GEO: mining millions of expression profiles--database and tools. Nucleic Acids Res 2005, 33 Database Issue:D562-6.

5. Parkinson H, Sarkans U, Shojatalab M, Abeygunawardena N, Contrino S, Coulson R, Farne A, Lara GG, Holloway E, Kapushesky M, Lilja P, Mukherjee G, Oezcimen A, Rayner T, Rocca-Serra P, Sharma A, Sansone S, Brazma A: ArrayExpress--a public repository for microarray gene expression data at the EBI. Nucleic Acids Res 2005, 33 Database Issue:D553-5.

6. Irizarry RA, Warren D, Spencer F, Kim IF, Biswal S, Frank BC, Gabrielson E, Garcia JG, Geoghegan J, Germino G, Griffin C, Hilmer SC, Hoffman E, Jedlicka AE, Kawasaki E, Martinez-Murillo F, Morsberger L, Lee H, Petersen D, Quackenbush J, Scott A, Wilson M, Yang Y, Ye $\mathrm{SQ}, \mathrm{Yu} \mathrm{W}$ : Multiple-laboratory comparison of microarray platforms. Nat Methods 2005, 2(5):345-350.

7. Wheeler DL, Barrett T, Benson DA, Bryant SH, Canese K, Church DM, DiCuccio M, Edgar R, Federhen S, Helmberg W, Kenton DL, Khovayko O, Lipman DJ, Madden TL, Maglott DR, Ostell J, Pontius JU, Pruitt KD, Schuler GD, Schriml LM, Sequeira E, Sherry ST, Sirotkin K, Starchenko G, Suzek TO, Tatusov R, Tatusova TA, Wagner L, Yaschenko E: Database resources of the National Center for 
Biotechnology Information. Nucleic Acids Res 2005, 33 Database Issue:D39-45.

8. Ball CA, Awad IA, Demeter J, Gollub J, Hebert JM, Hernandez-Boussard T, Jin H, Matese JC, Nitzberg M, Wymore F, Zachariah ZK, Brown PO, Sherlock G: The Stanford Microarray Database accommodates additional microarray platforms and data formats. Nucleic Acids Res 2005, 33 Database Issue:D580-2.

9. Manduchi E, Grant GR, He H, Liu J, Mailman MD, Pizarro AD, Whetzel PL, Stoeckert CJ]: RAD and the RAD Study-Annotator: an approach to collection, organization and exchange of all relevant information for high-throughput gene expression studies. Bioinformatics 2004, 20(4):452-459.

10. Ikeo K, Ishi-i J, Tamura T, Gojobori T, Tateno Y: CIBEX: center for information biology gene expression database. C $R$ Biol 2003, 326(I 0-I I): I079-1082.

II. Forster T, Roy D, Ghazal P: Experiments using microarray technology: limitations and standard operating procedures. J Endocrinol 2003, 178(2): 195-204.

12. Tseng GC, Oh MK, Rohlin L, Liao JC, Wong WH: Issues in cDNA microarray analysis: quality filtering, channel normalization, models of variations and assessment of gene effects. Nucleic Acids Res 200I, 29( I 2):2549-2557.

13. Dudoit S, Yang YH: Bioconductor $\mathbf{R}$ packages for exploratory analysis and normalization of cDNA microarray data . In The Analysis of Gene Expression Data: Methods and Software Edited by: Parmigiani IG, Garrett ES, Irizarry RA, Zeger SL. New York , Springer; 2003:73-10I.

14. Leung YF, Cavalieri D: Fundamentals of cDNA microarray data analysis. Trends Genet 2003, 1 9(I I):649-659.

15. Dobbin K, Shih JH, Simon R: Questions and answers on design of dual-label microarrays for identifying differentially expressed genes. J Natl Cancer Inst 2003, 95( I8): I362-1369.

16. Glasel JA: Validity of nucleic acid purities monitored by $260 \mathrm{~nm} / 280 \mathrm{~nm}$ absorbance ratios. Biotechniques 1995, 18(1):62-63

17. Goryachev AB, Macgregor PF, Edwards AM: Unfolding of microarray data. J Comput Biol 200I, 8(4):443-46I.

18. Lee ML, Kuo FC, Whitmore GA, Sklar J: Importance of replication in microarray gene expression studies: statistical methods and evidence from repetitive cDNA hybridizations. Proc Natl Acad Sci U S A 2000, 97(18):9834-9839.

19. Gentleman RC, Carey V], Bates DM, Bolstad B, Dettling M, Dudoit S, Ellis B, Gautier L, Ge Y, Gentry J, Hornik K, Hothorn T, Huber W, lacus S, Irizarry R, Leisch F, Li C, Maechler M, Rossini AJ, Sawitzki G, Smith C, Smyth G, Tierney L, Yang JY, Zhang J: Bioconductor: open software development for computational biology and bioinformatics. Genome Biol 2004, 5(I0):R80.

20. Lang R, Pauleau AL, Parganas E, Takahashi Y, Mages J, Ihle JN, Rutschman R, Murray PJ: SOCS3 regulates the plasticity of gp I 30 signaling. Nat Immunol 2003, 4(6):546-550.

21. Popkin DL, Watson MA, Karaskov E, Dunn GP, Bremner R, Virgin HW: Murine cytomegalovirus paralyzes macrophages by blocking IFN gamma-induced promoter assembly. Proc Nat Acad Sci U S A 2003, 100(24): |4309-|43|4.

22. Hoffmann R, van Erp K, Trulzsch K, Heesemann J: Transcriptional responses of murine macrophages to infection with Yersinia enterocolitica. Cell Microbiol 2004, 6(4):377-390.

23. Liu B, Mink S, Wong KA, Stein N, Getman C, Dempsey PW, Wu H, Shuai K: PIASI selectively inhibits interferon-inducible genes and is important in innate immunity. Nat Immunol 2004, 5(9):89।-898.

24. Tassiulas I, Hu X, Ho H, Kashyap Y, Paik P, Hu Y, Lowell CA, Ivashkiv LB: Amplification of IFN-alpha-induced STATI activation and inflammatory function by Syk and ITAM-containing adaptors. Nat Immunol 2004, 5(I I): I I8I-I I89.

25. Murray RZ, Wylie FG, Khromykh T, Hume DA, Stow JL: Syntaxin 6 and Vtilb form a novel SNARE complex, which is up-regulated in activated macrophages to facilitate exocytosis of tumor necrosis Factor-alpha. J Biol Chem 2005, 280( I I ): I0478-I0483.

26. Chaussabel D, Semnani RT, McDowell MA, Sacks D, Sher A, Nutman TB: Unique gene expression profiles of human macrophages and dendritic cells to phylogenetically distinct parasites. Blood 2003, 102(2):672-68I.
27. Leong HS, Yates T, Wilson C, Miller CJ: ADAPT: a database of affymetrix probesets and transcripts. Bioinformatics 2005, 2 I ( I 0):2552-2553.

28. Liu G, Loraine AE, Shigeta R, Cline M, Cheng J, Valmeekam V, Sun S, Kulp D, Siani-Rose MA: NetAffx: Affymetrix probesets and annotations. Nucleic Acids Res 2003, 3 I (I):82-86.

29. Maglott D, Ostell J, Pruitt KD, Tatusova T: Entrez Gene: genecentered information at NCBI. Nucleic Acids Res 2005, 33(Database issue):D54-8.
Publish with Biomed Central and every scientist can read your work free of charge

"BioMed Central will be the most significant development for disseminating the results of biomedical research in our lifetime. "

Sir Paul Nurse, Cancer Research UK

Your research papers will be:

- available free of charge to the entire biomedical community

- peer reviewed and published immediately upon acceptance

- cited in PubMed and archived on PubMed Central

- yours - you keep the copyright
BioMedcentral 